\title{
A Rare Complication of Diagnostic Angiography in a Child with Vertebro-Venous Fistula: Transient Total Cortical Blindness
}

\author{
Abdullah Erdem, Celal Akdeniz, Ender Ödemiş, Ahmet Çelebi \\ Department of Pediatric Cardiology, Dr. Siyami Ersek Thoracic and Cardiovascular Surgery Training and Research Hospital, i̇stanbul, Turkey
}

\begin{abstract}
Transient cortical blindness is a rare and dramatic complication after coronary angiocardiography in adulthood. This potential complication of transcatheter procedures which are widely used in the diagnosis and treatment of congential heart disease has not been reported during childhood. Although the exact mechanism is not fully understood, the direct idiosyncratic neurotoxicity is the most reasonable explanation. In this manuscript, we present a nine-year-old child who developed transient cortical blindness following diagnostic angiography with vertebro- venous fistula (WF) which might be the cause of this complication. In regard to this case, the pathogenetic mechanisms and management of this rare complication were also discussed.
\end{abstract}

Key Words: Angiography, transient total cortical blindness, child

Received: 11.05.2009

Accepted: 13.06 .2009

\section{Introduction}

Transient cortical blindness as a rare complication of angiocardiography has been well described in adults $(1,2)$. Contrast media penetration of the blood brain barrier, direct neurotoxic effect selectively to the occipital cortex, arterial spasm, allergic reaction to contrast media, and direct injection to the vertebral artery all claimed to explain this self limited, reversible phenomenon, but the mechanism is not yet completely understood $(3,4)$. We describe a nine-yearold child with vertebro-venous fistula (VVF) who experienced transient blindness after diagnostic angiography.

\section{Case Report}

A nine-years-old $24 \mathrm{~kg}$, asymptomatic boy with a continuous murmur in the right cervical region was referred for further evaluation. On physical examination there was a 2/6 continuous murmur best heard at right upper sternal border and right cervical region. The electrocardiogram and chest $\mathrm{x}$-ray were within normal limits. Echocardiographic examination revealed normal intracardiac anatomy and function, but evidence of a turbulent, high velocity flow suggested an arteriovenous malformation around the right carotid artery.

Angiographic evaluation was performed under general anesthesia in order to specify the diagnosis and possible transcatheter intervention at the same session. Arcus aortogram, selective carotid and vertebral arterial system angiography were performed. During the procedure, a total of $115 \mathrm{cc}(4.8 \mathrm{cc} / \mathrm{kg})$ of the non-ionic contrast media lopromid (Ultravist-370, Schering, Germany) containing $37 \mathrm{~g}$ of iodine in $100 \mathrm{cc}$ was used. Aortogram showed a large calibrated and tortuous brachiocephalic truncus, the right vertebral artery was also large calibrated, while both subclavian and common carotid arteries were normal in size. Early venous filling was noted in the right cervical region and superior vena cava. Selective right vertebral artery angiogram revealed a WV with an aneurysmal vertebral artery at the proximal part, communicating with a vein causing early filling of the cervical, radiculary and subclavian veins on both side, but prominent on the right (Figure 1). The distal part of the right vertebral artery and cerebellar arteries were hypoplastic. Filling of the cervical radiculary veins on both sides, more prominent on the right, was clearly delineated with the angiogram taken after advancing the catheter into the abnormal vein through the vertebral artery (Figure 2). Selective left subclavian artery angiogram revealed a dominant vertebral artery and a normal subclavian artery. Distal branching of the left vertebral artery was normal in the selective vertebral angiograms and reverse flow was not seen into the right vertebral artery from the basillary artery. Carotid arteries on both sides were within normal angiographic limits.

Soon after the patient recovered from the anesthesia, he was agitated and started to complain of severe headache and bilateral loss of vision. Emergency neurology and ophthalmology consultations were obtained. Neurological examination revealed no deficits and was found within normal limits. Both pupils were equal in size and reactive to light with no evidence of nystagmus. Ophthalmologic examination was consistent with bilateral loss of vision; however there was no evidence of overt abnormal physical examination findings. Blood counts, bleeding parameters, blood electrolytes were all within normal limits. Cranial MR with T1, T2, proton 


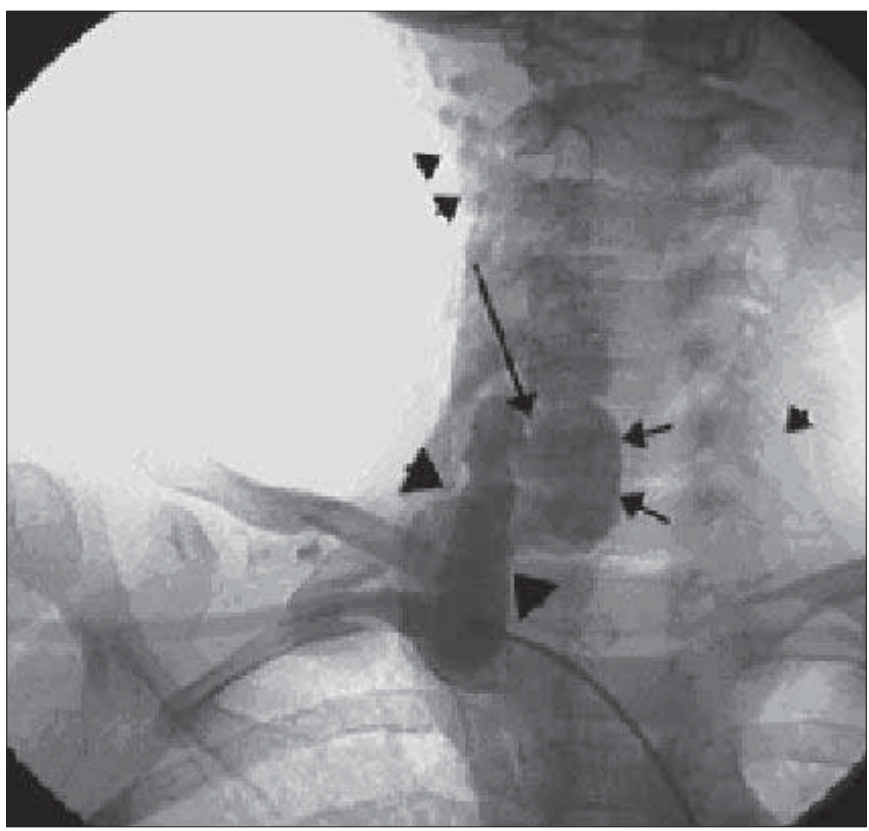

Figure 1. Selective right subclavian artery angiogram shows ectatic vertebral artery (large arrow heads) connecting (long arrow) with the aneurysmatic vein. Also note the ipsilateral and contralateral fillings of the cervical veins (small arrow heads)

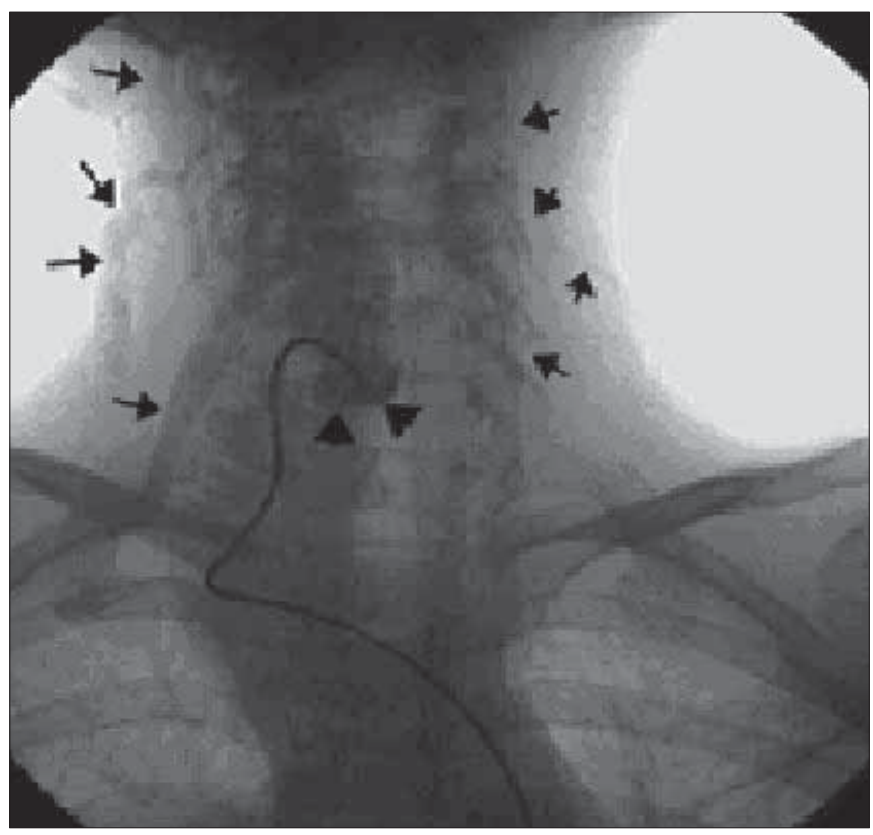

Figure 2. Angiogram taken after the placement of catheter into the vein (arrow heads), through the vertebral artery clearly demonstrates the filling of the cervical (long tail arrows) and radicular (short tail arrows) veins

weighted, fluid-attenuated inversion recovery (FLAIR) and diffusion studies were performed and all revealed normal results. There was no clinical evidence of an embolic event and no abnormal contrast enhancement in any part of the brain parenchyma. The patient was started on a heparin drip (20 IU/ $\mathrm{kg} / \mathrm{h}$ infusion after $100 \mathrm{lU} / \mathrm{kg}$ bolus injection) and intravenous dexamethasone treatment $(0.6 \mathrm{mg} / \mathrm{kg} /$ day divided into four doses) empirically. He dramatically recovered fully after 24 hours follow up. He was monitored for one more night and discharged without any sequel.

\section{Discussion}

Vertebro-venous fistula is a rare form of arteriovenous fistula and is defined as an abnormal communication between the vertebral artery, or its branches, and an adjacent venous system, usually the internal jugular vein or internal vertebral venous plexus (5). They are rare in children and the congenital form has seldom been reported in the literature (6). They are most commonly seen secondary to trauma and are more common in adults (6). Their clinical presentation ranges from tinnitus to overt ischemic posterior fossa signs, such as hemiparesis and quadriparesis, cortical blindness, and disturbances of wake-sleep rhythm (6). They may be asymptomatic, especially in children, and diagnosed incidentally by a bruit in the cervical or retromastoid regions (7). WVFs tend to enlarge over time by recruiting additional feeding arteries and increasing blood shunting, resulting in transient ischemic attacks and stroke in the vertebrobasilar territory. Progressively increased shunting can also cause cardiomegaly and high-output congestive heart failure (7). The prognosis is influenced by the patient's age at the time of diagnosis; generally cardiac complications predict the prognosis in pediatric patients, whereas neurological complications are more frequent in adults (7).

Intra-arterial angiography is essential in delineating the fistula, its supplying arteries and draining veins, mapping the route for procedures, establishing the degree of blood shunting, and in excluding the possible origin of a spinal artery from the tract to be embolised. Nevertheless, complications after angiography itself like vascular injury, peripheral emboli, infection, bleeding and arrhythmia and adverse reactions to contrast media are well described (8). Cortical blindness after contrast media exposure has been reported to be as high as $1-4 \%$ with vertebral angiography, but has only been described in a few cases of coronary angiography with modern non-ionic, low-osmolality contrast agents (1).Patients with WVF have chronic vertebrobasillary insufficiency. This phenomenon leads to chronic cerebral ischemia. Therefore, WV has a tendency to cause cerberovascular accidents (5). In our opinion, direct injection in vertebral artery and the presence of vertebro-venous fistula may be an additional cause of transient blindness in the presented case.

Contrast media penetration of the blood brain barrier and a direct selective neurotoxic effect to the occipital cortex, arterial spasm, allergic reaction to contrast media, and direct injection to the vertebral artery are all claimed to explain this self limited, reversible phenomenon but the mechanism is not yet completely understood $(2,3)$. Saigal et al. reported this phenomenon to be closely related to posterior reversible leukoencephalopathy and might have the same pathophysiology (9).

The blood-brain barrier may be permeable under the clinical settings of uncontrolled hypertension, renal insufficiency and use of immunosuppressive drugs. Angiographic contrast media tend to breach the blood-brain barrier of the vertebrobasilar circulation, penetrating the occipital cortex and lead- 
ing to transient cortical blindness or abnormal visuospatial processing disorders in such patients $(3,4)$. However, in our case there was no clinical inclination to increased blood brain barrier permeability or any suggestive findings and MRI findings were normal.

Most cases of cortical blindness resolve spontaneously within an hour to a few days, and no therapy has been proven to be effective (3). Our patient was treated with glucocorticoids, suggesting that steroid treatment may stabilize the blood-brain barrier and decrease radiocontrast induced immunological reactions. Although MRI findings showed no morphological changes indicative of embolic infarction, heparin was started in order to prevent micro thromboembolic events. The patient recovered completely after 24 hours. Both heparin and steroid treatments were withdrawn.

To our knowledge, the incidence and significance of transient cortical blindness after cerebral or cardiac angiography in the pediatric population has not been reported. Mentzel HJ et all reported a 16 year-old boy with diabetes insipidus and hyponatremia who experienced transient cortical blindness following contrast enhanced cranial CT (10). In that reported case the patient's symptoms were thought to be triggered by hyponatremia and diabetes insipidus. Our patient had normal serum electrolytes.

To our knowledge, this is the first reported case of transient cortical blindness in the pediatric population as a complication of angiography. This rare, but well known complication of cerebral angiography in adults may also be seen in the pediatric population. It was self limited and resolved spontaneously within hours to a few days, as reported in adults. Pediatric interventional cardiologists and radiologists involved with angiography in children should be aware of this rare but dramatic and also self limited phenomenon. They also should keep in mind that, in a vascular malformation which leads to a tendency to cerebrovascular events such as WVF, direct injection which results in increased contrast media concentration in the central cortical system may cause this rare complication, especially when patients have a vascular malformation such as WVF.

\section{Conflict of Interest}

No conflict of interest was declared by the authors.

\section{References}

1. Parry R, Rees JR, Wilde P. Transient cortical bilndness after coronary anigography. Br Heart J 1993;70:563-4 [CrossRef]

2. Studdard WE, Davis DO, Young SW. Cortical blindness after cerebral angiography. Case report. J Neurosurg 1981; 54:240-4. [CrossRef]

3. Lantos G. Cortical blindness due to osmotic disruption of the blood-brain barrier by angiographic contrast material: CT and MRI studies. Neurology 1989;39:567-71

4. Merchut MP, Richie B. Transient visuospatial disorder from angiographic contrast. Arch Neurol 2002;59:851-4. [CrossRef]

5. Taylor CG, Husami Y, Colquhoun IR, Byrne JV. Direct cervical vertebro-venous fistula with radiculopathy and MRI changes resolving after successful endovascular embolisation: a report of two cases. Neuroradiology 2001;43:1118-22.

6. Shownkeen H, Bova D, Chenelle AG, Origitano TC. Vertebral arteriovenous fistulas. Clinical presentation, angiographical appearance and endovascular treatment. A review of twenty-five cases. Ann Radiol 1985;28:425-38.

7. Shownkeen H, Bova D, Chenelle AG, Origitano TC. Pediatric congenital vertebral artery arteriovenous malformation. Pediatr Radiol 2003;354-6.

8. Stanger P, Heymann MA, Tarnoff $\mathrm{H}$, Hoffman JI, Rudolph AM. Complications of cardiac catheterization of neonates, infants, and children. A three-year study. Circulation 1974;50:595-608.

9. Saigal G, Bhatia R, Bhatia S, Wakhloo AK. MR findings of cortical blindness following cerebral angiography: is this entity related to posterior reversible leukoencephalopathy? AJNR 2004;25:252-6.

10. Mentzel HJ, Blume J, Malich A, Fitzek C, Reichenbach JR, Kaiser WA. Cortical blindness after contrast-enhanced CT: complication in a patient with diabetes insipidus. AJNR 2003;24:1114-6. 